\title{
Predictive energy management for hybrid electric vehicles - Prediction horizon and battery capacity sensitivity
}

\author{
M. Debert ${ }^{*, * *}$ G. Colin ${ }^{*}$ Y. Chamaillard ${ }^{*}$ L. Guzzella ${ }^{* *}$ \\ A. Ketfi-Cherif ${ }^{* * *}$ B. Bellicaud ${ }^{*}$ \\ * University of Orleans, Orleans, France (e-mail: \\ maxime.debert@renault.com). \\ ** ETHZ, Zurich, Switzerland. \\ *** Renault, Guyancourt, France .
}

\begin{abstract}
Increasing information about future driving conditions in vehicles makes predictive energy management realistic. This energy management strategy uses a dynamic programming algorithm on a sliding window in order to minimize the hybrid vehicle fuel consumption. For real time implementation, it is necessary to reduce computational time so as to embed this control on an automotive calculator. This paper focuses on the influence of the prediction horizon and battery capacity on $\mathrm{CO}_{2}$ emission in the case of a combined hybrid electric vehicle.
\end{abstract}

Keywords: Hybrid vehicles, Energy Management, Predictive control, Optimal control, Dynamic programming, Battery

\section{INTRODUCTION}

The hybrid vehicle is one of the possible solutions for reducing greenhouse gas emission in passenger transportation. The opportunity for using different prime movers to satisfy the power demand allows the supervisory control to choose the energy flow that minimizes global greenhouse gas emission. This optimization problem, often called energy management (Guzzella and Sciarretta (2007)), has sparked the interest of researchers (Sciarretta and Guzzella (2007)) and car manufacturers because it enhances fuel economy without increasing the final cost of the vehicle. When the entire mission is known, optimization algorithms such as dynamic programming (Bertsekas (2005)) can find the optimal power flow. Since however, in real-time implementation, the mission is unknown at the outset, dynamic programming cannot be used.

The estimation of future driving conditions on a sliding window will become possible with improvements in telematics and new radar system, leading some authors (Back et al. (2002), Koot et al. (2004)) to develop predictive energy management. This control strategy estimates future power demand with a vehicle model and then uses dynamic programming on this estimation. The strategy respects the model predictive control (MPC) principle (Morari and Lee (1999), Qin and Badgwell (2003)), initially developed for low dynamical system dynamic systems such as the process industries.

This paper proposes to study the influence of the prediction horizon and battery capacity on fuel economy. While it is logical that fuel consumption will decrease with an increase in the prediction horizon, it is not straightforward to determine to what extent this results in fuel economy. Battery capacity was studied for optimal sizing in
(Rousseau et al. (2008), Sundstrom et al. (2008)). In this study, the purpose is to understand the influence of battery capacity for a predictive strategy. An illustration on a dual-mode power-split hybrid powertrain (Debert et al. (2010)) is proposed. The hybrid transmission is composed of a planetary gear sets arrangement and two electric motors (Villeneuve (2004)).

The first section describes the hybrid vehicle and its modeling with a focus on the battery model. The second section is devoted to the predictive energy management formulation. The third section presents some results and interpretations.

\section{THE HYBRID VEHICLE MODEL}

\subsection{The hybrid powertrain}

The hybrid vehicle studied uses a complex transmission composed of planetary gear sets and two electric motors connected to a power battery Fig. 1. This transmission has a dual-mode structure, with the first mode used for low speed and the second mode for high speed. The dualmode structure enhances power passing through the high efficiency mechanical path, thereby improving the global efficiency of the powertrain (Debert et al. (2010)).

The predictive energy management strategy (Back et al. (2002)) is based on a quasi-static vehicle model. This model evaluates $\mathrm{CO}_{2}$ emission and the variation of battery state of charge as a function of control inputs and vehicle speed and acceleration.

\subsection{Quasi-static model}

From the vehicle speed and acceleration a longitudinal dynamic model of the vehicle gives the wheel torque $T_{o}$ 


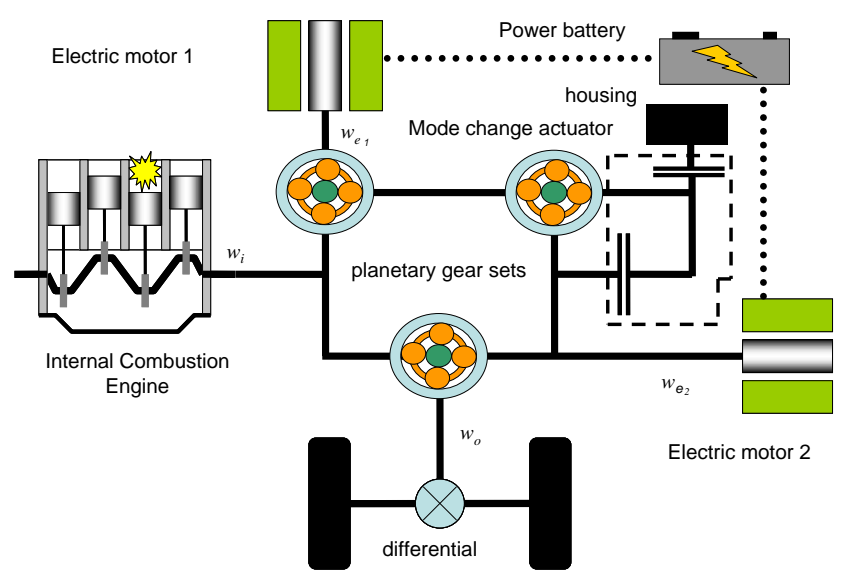

Fig. 1. Hybrid powertrain configuration

and wheel speed $w_{o}$ (Guzzella and Sciarretta (2007)). Since power losses in the kinematic chain are negligible, the energy conservation gives

$$
P_{e}+T_{i} w_{i}+T_{o} w_{o}=0,
$$

where $T_{i}$ and $w_{i}$ represent respectively the engine torque and speed. $P_{e}$ represents mechanical power provided or recuperated by the two electric motors

$$
P_{e}=T_{e_{1}} w_{e_{1}}+T_{e_{2}} w_{e_{2}},
$$

with $T_{e_{1}}, T_{e_{2}}, w_{e_{1}}, w_{e_{2}}$ the torque and speed of electric motor 1 and 2 .

To realize drivers' demands which are interpreted as a wheel torque $T_{o}$ for a given wheel speed $w_{o}$, the system has two degrees of freedom (1). This assertion is well known for a combined hybrid configuration Sciarretta and Guzzella (2007). The predictive energy management strategy, discussed in the following section, makes the most of these degrees of freedom for reducing $\mathrm{CO}_{2}$ emission. Since $T_{o}, w_{o}$ are already known in (1), the energy management can set two manipulated variables chosen among the three variables $T_{i}, w_{i}, P_{e}$. For reasons of representativity, the commonly manipulated variable for internal combustion engine torque $T_{i}$ and the power $P_{e}$ provided or recuperated in the kinematic chain by the electrical path were chosen. Thus the control vector is denoted

$$
\mathbf{u}=\left[\begin{array}{l}
T_{i} \\
P_{e}
\end{array}\right]
$$

Then the engine speed $w_{i}$ is calculated considering the energy conservation in the kinematic chain (1)

$$
w_{i}=-\frac{T_{o} w_{o}+P_{e}}{T_{i}} .
$$

The engine fuel consumption is computed with a map obtained from engine test bench and the $\mathrm{CO}_{2}$ emission is deduced using the fuel properties.

Then the electrical path set points are also calculated using the kinematic relations of the electric variable transmission (5) and (6).

$$
\begin{aligned}
& \text { Mode 1: }\left(\begin{array}{c}
w_{i} \\
w_{o}
\end{array}\right)=\left[\begin{array}{ll}
A_{1} & B_{1} \\
C_{1} & D_{1}
\end{array}\right] \cdot\left(\begin{array}{l}
w_{e_{1}} \\
w_{e_{2}}
\end{array}\right)=M_{1} \cdot\left(\begin{array}{l}
w_{e_{1}} \\
w_{e_{2}}
\end{array}\right) \\
& \text { Mode 2: }\left(\begin{array}{c}
w_{i} \\
w_{o}
\end{array}\right)=\left[\begin{array}{ll}
A_{2} & B_{2} \\
C_{2} & D_{2}
\end{array}\right] \cdot\left(\begin{array}{l}
w_{e_{1}} \\
w_{e_{2}}
\end{array}\right)=M_{2} \cdot\left(\begin{array}{l}
w_{e_{1}} \\
w_{e_{2}}
\end{array}\right) .
\end{aligned}
$$

the coefficients $A_{1}, A_{2}, B_{1}, B_{2}, C_{1}, C_{2}, D_{1}, D_{2}$ are fixed by the planetary gear set design (i.e. by the number of teeth in each component).

$$
\text { Mode } 1:\left(\begin{array}{l}
T_{i} \\
T_{o}
\end{array}\right)=M_{1}^{T} \cdot\left(\begin{array}{l}
T_{e_{1}} \\
T_{e_{2}}
\end{array}\right)
$$

\subsection{Battery model}

The battery represents a reversible electrical energy storage system; the amount of charge remaining in the battery is the state of charge, expressed as a percentage of the battery nominal capacity. Since the battery state of charge is the unknown result of energy management control, this variable has to be modeled as a dynamic system.

Power provided or recuperated by the battery is calculated by adding up all the electrical power demands

$$
\begin{aligned}
P_{b a t}= & T_{e_{1}} w_{e_{1}}+P_{{l o s s_{1}}_{1}}\left(T_{e_{1}}, w_{e_{1}}, U_{b a t}\right)+ \\
& T_{e_{2}} w_{e_{2}}+P_{l_{\text {los }}}\left(T_{e_{2}}, w_{e_{2}}, U_{b a t}\right)+P_{a c c}
\end{aligned}
$$

with $P_{l o s s_{1}}, P_{\text {loss }_{2}}$ the power losses in the electric motors. These losses depend on the electric motors set points given by (5),(6) and battery voltage $U_{b a t}$ (i.e. the electric motors are directly linked to the battery). $P_{a c c}$ represents the power consumption of all auxiliary electrical loads.

The battery is then modeled as an open circuit voltage source $o c v$ in series with an internal resistance $R_{\text {int }}$. These two components vary with battery state of charge $x$. Battery current $I_{b a t}$ is calculated from Kirchhoff's law

$$
I_{b a t}=\frac{o c v(x)-\sqrt{o c v(x)^{2}-4 P_{b a t} R_{i n t}(x)}}{2 R_{\text {int }}(x)} .
$$

Battery state of charge, representing the dynamic part of the model, is calculated by integrating the current (9). In the case of energy recuperation a fraction of the current is not transformed into charge. This physical loss is modeled by the Coulombic efficiency $\eta$

$$
\begin{aligned}
& x(t)=\frac{1}{Q_{\max }} \int_{0}^{t} I_{b a t}^{*}(\tau) d \tau \\
& I_{b a t}^{*}=\left\{\begin{array}{lll}
I_{b a t} & \text { if } & I_{b a t} \geq 0 \\
\eta I_{b a t} & \text { if } & I_{b a t}<0,
\end{array}\right.
\end{aligned}
$$

where $Q_{\max }$ is the maximum charge capacity of the battery. Because the purpose of this paper is to understand the influence of battery capacity, $Q_{\max }$ varies according to the experiment. Then the state dynamic which represents the variation in the battery state of charge is described by

$$
\dot{x}(t)=f(x(t), \mathbf{u}(t)), \quad x(0)=x_{0} .
$$

As the battery current is correlated to thermal dissipation by the Joule effect, the lower the battery current, the higher the battery efficiency. For a given battery power, a high open circuit voltage source ensures low current (8). Open circuit voltage is a function of battery state of charge. Since the battery state of charge dynamics depends on battery capacity (9), it is logical to study this influence on the overall efficiency. The studied battery capacity is border between 0.8 to $6.3 \mathrm{kWh}$, considering $100 \mathrm{Wh} / \mathrm{kg}$, the influence of battery capacity on the overall vehicle mass was neglected. 


\section{PREDICTIVE ENERGY MANAGEMENT}

\subsection{Energy management general formulation}

The energy management controller has to set the two manipulated variables $\mathbf{u}=\left[T_{i}, P_{e}\right]^{T}$ throughout the entire mission in order to minimize $\mathrm{CO}_{2}$ emission. This problem is equivalent to a dynamic constrained optimization problem

$$
\left\{\begin{array}{l}
\min _{u}\left\{J(x, \mathbf{u})=\int_{0}^{T} L(\mathbf{u}(t)) d t+\Phi(x(T))\right\} \\
\text { with respect to : } \\
\dot{x}(t)=f(x(t), \mathbf{u}(t)), \quad x(0)=x_{0} \\
x(t) \in X, \forall t \geq 0 \\
\mathbf{u}(t) \in U, \forall t \geq 0 .
\end{array}\right.
$$

with $\Phi(x(T))$ a penalty function which guarantees a minimum battery state of charge at the end of the mission, $L(\mathbf{u}(t))$ an instantaneous greenhouse gas emission. $\mathbf{u}(t)$ represents the control vector and $x(t)$ the state variable which represents battery state of charge. Furthermore the input and state constraints sets $U, X$ are defined by

$$
\begin{aligned}
& U=\left\{\mathbf{u} \in \mathbb{R}^{2} \mid \mathbf{u}_{\min }(t) \leq \mathbf{u} \leq \mathbf{u}_{\max }(t)\right\} \\
& X=\left\{x \in \mathbb{R} \mid x_{\min } \leq x \leq x_{\max }\right\}
\end{aligned}
$$

$x_{\text {min }}, x_{\text {max }}$ are physical battery state of charge limitations $\mathbf{u}_{\min }, \mathbf{u}_{\max }$ are input constraints such as the engine and electric motors limitations. It is also possible to include for example engine limitations for low speed drive in order to enhance noise agreement. Since in real time implementation, the entire mission cannot be known at the outset, this optimization problem cannot be solved directly. One way to minimize the overall $\mathrm{CO}_{2}$ emission is to solve the previous problem on a prediction of future driving conditions.

\subsection{Predictive control}

The control used in this paper follows the model predictive control paradigm. This predictive control is formulated as a repeated solution of a finite horizon optimal control problem with respect to system dynamics, input and state constraints. For each sampling period of the energy management calculator the following sequence is repeated:

- Generation of the future trajectory

- Minimization of the cost function over the receding prediction horizon starting with the current state of charge

- Application of the first part of the control signal on the process during the sampling period of the calculator.

At the computation time $t_{0}$, the future trajectory $\hat{T}_{o}(\tau)$, $\hat{w}_{o}(\tau), \tau \in\left[t_{0} ; t_{0}+t_{p}\right]$ on the prediction horizon $t_{p}$ is estimated with onboard instruments and model based algorithm. For this study, the estimation is a perfect representation of future driving conditions.

Then a dynamic programming algorithm finds the optimal control $\mathbf{u}(\tau)$ that minimizes greenhouse gas emission on the future trajectory $\hat{T}_{o}(\tau), \hat{w}_{o}(\tau)(13)$.

$$
\left\{\begin{array}{l}
\min _{u}\left\{J(\hat{x}, \mathbf{u})=\int_{t_{0}}^{t_{0}+t_{p}} \hat{L}(\mathbf{u}(t)) d t+\Theta\left(\hat{x}\left(t_{o}+t_{p}\right)\right)\right\} \\
\text { with respect to : } \\
\dot{\hat{x}}(t)=f(\hat{x}(t), \mathbf{u}(t)), \quad \hat{x}\left(t_{0}\right)=x\left(t_{0}\right) \\
\hat{x}(t) \in \aleph(t), \forall t \geq 0 \\
\mathbf{u}(t) \in U, \forall t \geq 0 .
\end{array}\right.
$$

The quasi-static model of the vehicle gives the estimation of greenhouse gas emission $\hat{L}$ and the variation of battery state of charge $\dot{\hat{x}}$ (Fig. 2).

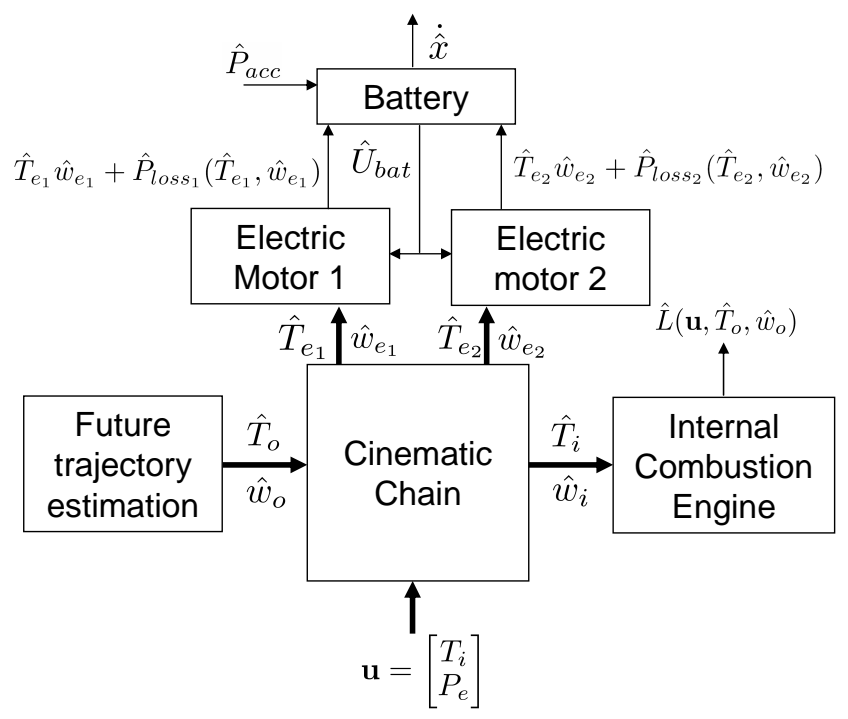

Fig. 2. Signal flow of the quasi-static model of the vehicle

As in Back et al. (2002), the penalty function is governed by (14) which guarantees charge sustaining but doesn't limit kinetic energy recuperation

$$
\Theta(x)=\left\{\begin{array}{lll}
0 & \text { if } & x \geq x_{\text {end }} \\
\infty & \text { if } & x<x_{\text {end }}
\end{array}\right.
$$

Where $x_{\text {end }}$ represents the minimum value of final battery state of charge.

\subsection{Computational time reduction}

The computational time required is the main disadvantage of the dynamic programming algorithm. In order to apply optimal predictive control during the sampling period, it is therefore necessary to introduce some improvements so as to reduce the computational burden.

The search grid $\aleph$ (Fig. 3) is reduced by considering the maximum energy recuperation or the maximum battery discharge capacity (Back et al. (2004))

$$
\aleph(t)=\left\{\hat{x}(t) \in \mathbb{R} \mid \bar{x}_{\text {min }}(t) \leq \hat{x}(t) \leq \bar{x}_{\text {max }}(t)\right\},
$$

$\bar{x}_{\text {min }}(t)$ and $\bar{x}_{\text {max }}(t)$ are defined considering the physical limitations of prime movers and battery

$$
\left\{\begin{array}{l}
\bar{x}_{\min }(t)=\max \left\{\hat{x}_{t_{0}+t_{p}}^{+}(t), \hat{x}_{t_{0}}^{-}(t), x_{\min }\right\} \\
\bar{x}_{\max }(t)=\min \left\{\hat{x}_{t_{0}}^{+}(t), x_{\max }\right\}
\end{array}\right.
$$

where $\hat{x}_{t_{0}+t_{p}}^{+}(t)$ represents the maximum rechargeable trajectory that respects the final battery state of charge constraint, $\hat{x}_{t_{0}}^{-}(t)$ is the maximum charge depleting trajectory for the starting battery state of charge $x_{t_{0}}$ and $\hat{x}_{t_{0}}^{+}(t)$ is the 


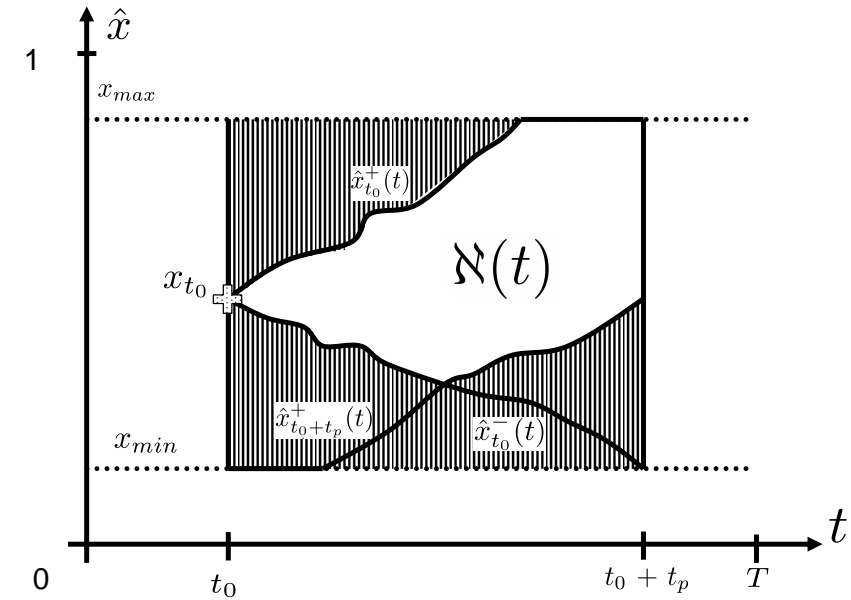

Fig. 3. Reduction of state space grid search

maximum rechargeable trajectory for the starting battery state of charge $x_{t_{0}}$.

Reducing the search grid is not enough for real time implementation because the optimization results require more time than the sampling period of the calculator. In Koot et al. (2005) the authors approximate the cost criterion with a second order polynomial function of the manipulated variable and then use quadratic programming to solve the optimization problem. While this method considerably reduces computational time, it is not suited for the combined hybrid energy management purpose since a second order polynomial function of manipulated variables and wheel set point $\left(T_{o}, w_{o}\right)$ is not representative of $\mathrm{CO}_{2}$ emission. The loss of information induced by this approximation results in a control strategy which is too far from optimal solution.

Another solution is to reduce the dynamic programming algorithm computational time by constraining some of the manipulated variables (Debert et al. (2010)). Considering that energy losses mainly come from the internal combustion engine, the strategy favors best engine efficiency set points by fixing the engine speed $w_{i}$ and engine torque $T_{i}$ as a function of the engine power $P_{i}$

$$
\left[T_{i}, w_{i}\right]=g\left(P_{i}\right) \text {. }
$$

Therefore the system is more constrained and a degree of freedom is lost which means that the previous multi input control is transformed into a single input control

$$
u=P_{i} \text {. }
$$

This strategy gives sub-optimal results but the increase in fuel consumption is acceptable for the predictive control strategy. Results presented in Debert et al. (2010) shows that for a NEDC cycle this strategy gives an extra fuel consumption of $3 \%$ with a computational time divided by 62 .

\subsection{Implementation}

The predictive energy management algorithm respects real time implementation requirements. Computational time was sufficiently reduced for the predictive energy management strategy to be used in a vehicle calculator. In Finkeldei and Back (2004) the MPC algorithm was embedded on a power PC.
A possible interaction of the predictive management in the vehicle control environment is proposed in Fig. 4. The control architecture is based upon four different layers.

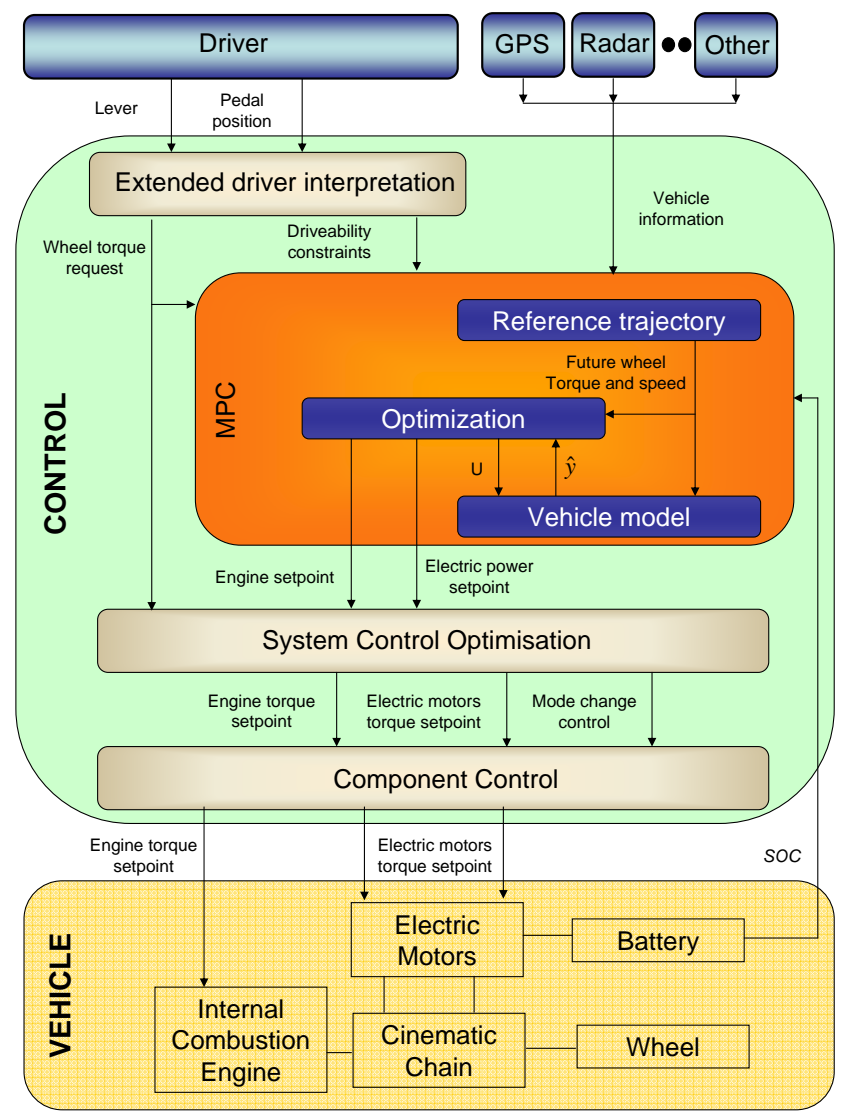

Fig. 4. Integration of control architecture with predictive energy management in the vehicle environment

- Extended Driver Interpretation is devoted to the interpretation of driver demands. It analyzes signals coming from driver actuators such as throttle pedal, gear shift lever, Zero Emission Vehicle button (i.e. which forces a purely electric drive) etc. and the vehicle environment (vehicle speed, road slope etc.). With this information, it calculates the required torque at the wheel and driveability constraints to ensure a smooth drive.

- MPC calculates the best prime mover parameters in terms of fuel consumption that satisfy driver demands. This layer integrates the predictive energy management algorithm.

- System Control Optimization ensures a dynamic control of prime mover parameters, mode change, engine stop-start, torque creeping etc. It implements, as well as possible, references coming from the operating point optimization layer.

- Component Control ensures dynamic control of components especially in transient modes.

This control architecture was tested on a dynamic model of the vehicle with a driver model. 


\section{RESULTS}

\subsection{Simulation}

This paper proposes to study the influence of the horizon prediction and the battery capacity on $\mathrm{CO}_{2}$ emission in a predictive energy management. The predictive energy management previously described was applied on different driving cycles with the same initial and final battery state of charge of $50 \%$ and $30 \%$. The charge sustaining around $30 \%$ represents the embedded strategy for a plug-in hybrid when the available electrical energy is low (i.e. often used for homologation strategies after the charge depleting). The charge sustaining around $50 \%$ represents the embedded strategy for conventional hybrid. An example of predictive strategy simulation is proposed on Fig. 5

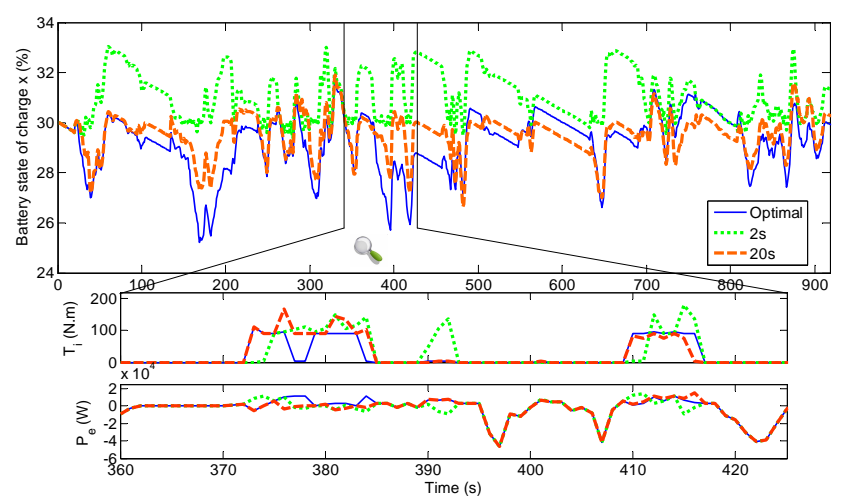

Fig. 5. State of charge trajectory and control inputs for optimal control and for predictive strategy with a prediction horizon of $2 \mathrm{~s}$ and 20s; Obtained on the Artemis urban driving cycle with a charge sustaining of $30 \%$

The final constraints (14) forces the predictive strategy to limits battery state of charge range. When the prediction horizon grows, the predictive strategy disposes of more liberty to wide this range. Fig. 5 shows that the battery state of charge trajectory for the $2 \mathrm{~s}$ predictive horizon is higher than the $20 \mathrm{~s}$ one. $2 \mathrm{~s}$ of prediction horizon is not enough to estimate kinetic energy recuperation while braking.

\subsection{Influence of prediction horizon}

Since the studied variable is the prediction horizon, the battery capacity is set at $1.3 \mathrm{kWh}$ which is the battery capacity of the Toyota Prius III. Because predictive energy management doesn't constraint free kinetic energy recuperation (14)(Fig. 5), final battery state of charge is not exactly the same for different prediction horizons. Therefore the final $\mathrm{CO}_{2}$ emission cannot be directly compared, but is compared to the optimal $\mathrm{CO}_{2}$ emission given by a dynamic programming algorithm (Debert et al. (2010))(i.e. DP is applied using, for each case, the same value of final SOC as the predictive strategy). Table 1 presents extra $\mathrm{CO}_{2}$ emission on standard regulatory cycle and on the Artemis driving cycles (Andre (2004)).

Since on-board information about future driving conditions is limited, prediction horizons higher than 30s are not considered. Moreover computational time increases with
Table 1. Extra $\mathrm{CO}_{2}$ emission in different driving cycles with a charge sustaining strategy around $30 \%$

\begin{tabular}{cccccc} 
Cycle / Horizon (s) & 2 & 5 & 10 & 20 & 30 \\
\hline Artemis Extra-urban & $4 \%$ & $2.6 \%$ & $2 \%$ & $1.8 \%$ & $1 \%$ \\
Artemis Urban & $6.6 \%$ & $5.2 \%$ & $4.5 \%$ & $2.3 \%$ & $1.2 \%$ \\
Artemis Traffic-Jam & $14.7 \%$ & $5.7 \%$ & $3.8 \%$ & $2.3 \%$ & $1.2 \%$ \\
NEDC & $3.1 \%$ & $2.3 \%$ & $2 \%$ & $1.5 \%$ & $1 \%$ \\
\hline
\end{tabular}

high predictive horizon which runs counter to real time implementation requirements.

As expected, the $\mathrm{CO}_{2}$ emission decreases when the predictive energy management possesses of more information about future driving conditions. The relation between $\mathrm{CO}_{2}$ emission and predictive horizon is not linear. Increasing predictive horizon from 2 seconds to 3 seconds is more interesting for reducing $\mathrm{CO}_{2}$ emission than increasing it from 20 seconds to 21 seconds. Considering the general form of extra $\mathrm{CO}_{2}$ emission $(Z)$ as a function of predictive horizon, it can be approximated by a decreasing exponential function

$$
Z=\alpha \cdot e^{-\beta t_{p}} .
$$

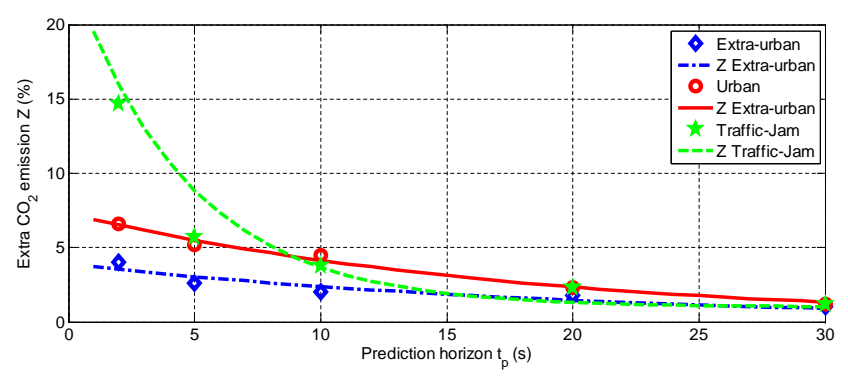

Fig. 6. Approximation of the influence of the prediction horizon $t_{p}$ on extra $\mathrm{CO}_{2}$ emission in different driving cycles

\subsection{Influence of battery capacity}

This section is devoted to the influence of battery capacity $Q_{\max }$ on fuel consumption in predictive energy management.

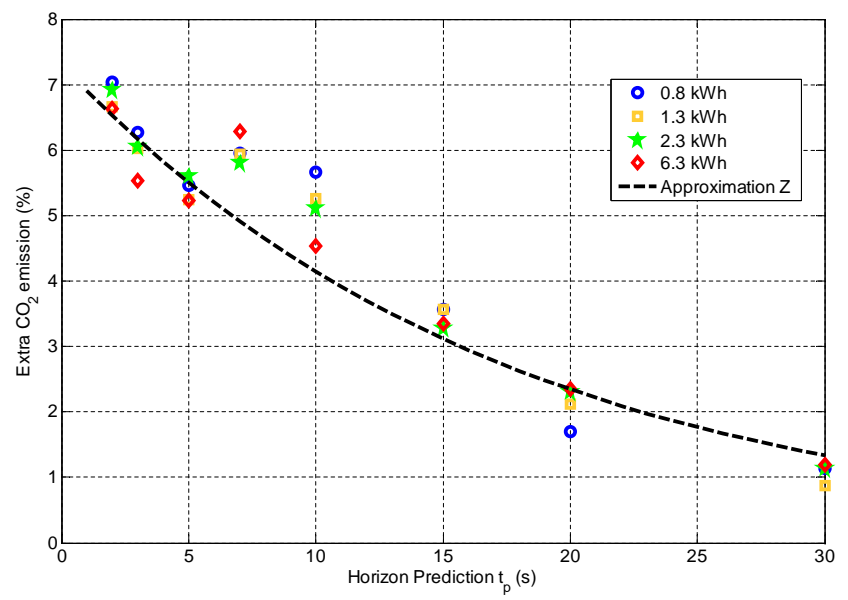

Fig. 7. Extra $\mathrm{CO}_{2}$ emission of predictive strategy in the Artemis Urban cycle for different battery capacities with a charge sustaining strategy around 50\% 
If the amount of energy available during recuperation events exceeds battery capacity, some energy cannot be recuperated and must be wasted in conventional braking. This event considerably decreases the predictive energy strategy performance and is not the purpose of this study. Therefore results presented in this section always transform kinetic energy into electrochemical energy with respects to battery state of charge limitations.

The predictive strategy with different battery capacities and predictive horizons was evaluated on standard regulatory cycles and on the Artemis cycle. An example of simulation result obtained on an Artemis Extra-urban driving cycle is proposed in Fig. 7. This result shows that battery capacity has no real impact on the performance of predictive strategy. In the studied hybrid configuration, the thermal engine operating point is completely independent of battery state of charge. Moreover the open circuit voltage variation in battery state of charge domain $X$ is relatively low for Li-ion battery technology. This explains the results obtained for all the driving cycles tested. Note that there are no energy losses in conventional braking which would penalize lower battery capacity.

Sometimes there is local increasing extra fuel consumption for higher prediction horizon (e.g. between $5 \mathrm{~s}$ and $7 \mathrm{~s}$ of prediction horizon). This can be explained by comparing the battery state of charge trajectory on the overall driving cycle. When this exceptional event occurs the trajectory taken by the lower prediction horizon is closer to the optimal one than the higher horizon.

\section{CONCLUSION}

Global optimizations such as dynamic programming are not directly suited for real time requirements. However, the future driving conditions can be predicted during real time operation, making it possible to use a model predictive control on the receding windows. The predictive control uses a dynamic programming algorithm applied on a vehicle model. Improvements are required to reduce computational time in order to use this strategy in real time. A classical optimization of state space search is necessary but not sufficient for a combined hybrid. A new strategy based on the engine efficiency, well suited for the hybrid configuration considered, was used and considerably reduces computational effort.

Simulation clearly demonstrated the benefits of a high predictive horizon on fuel consumption for the studied hybrid vehicle. Extra $\mathrm{CO}_{2}$ emission compared to the optimal solution as a function of the predictive horizon can be approximated as a decreasing exponential function. When there is no energy loss in conventional brakes the influence of battery capacity is negligible on the performance of the predictive strategy. For this hybrid configuration with the predictive energy management proposed, high battery capacity serves only to ensure autonomy in the purely electric drive range.

The influence of perturbations in future driving conditions in predictive energy management remains an open question. It would be interesting to know the impact of these perturbations on final $\mathrm{CO}_{2}$ emission.

\section{REFERENCES}

Andre, M. (2004). The artemis european driving cycles for measuring car pollutant emissions. Science of the total environment, 334-335, 73-84.

Back, M., Simons, M., Kirschaum, F., and Krebs, V. (2002). Predictive control of drivetrains. IFAC 15th triennial world congress, Barcelona Spain.

Back, M., Terwen, S., and Krebs, V. (2004). Predictive powertrain control for hybrid electric vehicles. Proc. IFAC Symposium on Advances in Automotive Control, University of Salerno, Italy.

Bertsekas, D. (2005). Dynamic programming and optimal control. Athena scientific.

Debert, M., Colin, G., Chamaillard, Y., Mensler, M., KetfiCherif, A., and Guzzella, L. (2010). Energy management of a high efficiency hybrid transmission. SAE Technical Paper, 2010, 2010-01-1311.

Finkeldei, E. and Back, M. (2004). Implementing an mpc algorithm in a vehicle with a hybrid powertrain using telematics as a sensor for powertrain control. IFAC Advances in automotive control, Salerno Italy.

Guzzella, L. and Sciarretta, A. (2007). Vehicle propulsion systems: introduction to modeling and optimization. Springer.

Koot, M., de Jager, B., Kessels, J., , Heemels, W., and van den Bosch, P. (2004). Energy management strategies for vehicle power nets. Proc. of the 2004 American control conference, Boston,USA.

Koot, M., Kessels, J., de Jager, B., Heemels, W., van den Bosch, P., and Steinbuch, M. (2005). Energy management strategies for vehicular electric power systems. IEEE transactions on vehicular technology, 54(3), 771782 .

Morari, M. and Lee, J. (1999). Model predictive control: past, present and future. Computers and chemical engineering, 23, 667-682.

Qin, S. and Badgwell, T. (2003). A survey of industrial model predictive control technology. Control engineering practice, 11, 733-764.

Rousseau, G., Sinoquet, D., Sciarretta, A., and Milhau, Y. (2008). Design optimisation and optimal control for hybrid vehicles. International conference on engineering optimization.

Sciarretta, A. and Guzzella, L. (2007). Control of hybrid electric vehicles - a survey of optimal energy management strategies. IEEE Control system magazine, 27(2), 60-70.

Sundstrom, O., Guzzella, L., and Soltic, P. (2008). Optimal hybridization in two parallel hybrid electric vehicles using dynamic programming. Proc. of the 17th IFAC World Congress, Seoul Korea.

Villeneuve, A. (2004). Dual mode electric infinitely variable transmission. Aachen Kolloquium Fahrzeug und Motorentechnik, 895-921. 